\title{
Thyroid storm after coronary artery bypass grafting
}

\author{
Lawrence T. Bish, PhD, Joseph E. Bavaria, MD, and John Augoustides, MD, FASE, FAHA, Philadelphia, Pa
}

We describe a 45-year-old woman with thyroid storm developing after urgent coronary artery bypass grafting (CABG). The hallmark clinical features included fever, tachycardia, depressed mental status, and jaundice. Thyroid testing confirmed severe hyperthyroidism caused by Graves disease. The patient was rendered euthyroid after initiation of therapy with methimazole (INN, thiamazole), potassium iodide, and hydrocortisone.

\section{CLINICAL SUMMARY}

A 45-year-old female recent immigrant from India came to the emergency department with a report of severe epigastric pain. Other than smoking, she had no history of coronary risk factors or previous cardiovascular disease. The patient took no regular medication and denied exposure to any tranquilizers. She was noted to be afebrile with a baseline sinus tachycardia of 114 beats/min and a blood pressure of $85 / 56 \mathrm{~mm} \mathrm{Hg}$. No goiter was detected at that time. Results of the rest of the physical examination were nonspecific. Her electrocardiogram showed anterior ST depression. Her serum troponin level was 10 times normal. Transthoracic echocardiography demonstrated mild global systolic dysfunction with an ejection fraction of $45 \%$ and mild global hypokinesis. Subsequent coronary angiography demonstrated significant stenoses of the left anterior descending and circumflex coronary arteries.

On the basis of these findings, the patient underwent urgent $\mathrm{CABG}$ with cardiopulmonary bypass. The patient was not febrile intraoperatively. The maximal patient temperature during surgery was $36.8^{\circ} \mathrm{C}$. The 2 coronary artery grafts were a left internal thoracic artery to the left anterior descending coronary artery and a saphenous vein graft from the ascending aorta to the circumflex coronary artery.

Intraoperative transesophageal echocardiography demonstrated severe left ventricular systolic dysfunction, with an ejection fraction of $20 \%$, as the result of hypokinesis of the septum, apex, and anterior wall. The working diagnosis, in light of the echocardiographic findings, for the new left ventricular dysfunction after separation from cardiopulmonary

\footnotetext{
From the University of Pennsylvania School of Medicine, Philadelphia, Pa.

Supported by Anesthesiology and Critical Care, University of Pennsylvania School of Medicine, Philadelphia, Pa.

Disclosures: None.

Received for publication May 5, 2010; revisions received June 15, 2010; accepted for publication June 29, 2010; available ahead of print Aug 9, 2010.

Address for Reprints: John Augoustides, MD, FASE, FAHA, Associate Professor, Cardiovascular and Thoracic Section, Anesthesiology and Critical Care, 680 Dulles, University of Pennsylvania Medical Center, 3400 Spruce St, Philadelphia, PA 19104-4283 (E-mail: yiandoc@hotmail.com).

J Thorac Cardiovasc Surg 2010;140:e67-9

$0022-5223 / \$ 36.00$

Copyright $₫ 2010$ by The American Association for Thoracic Surgery

doi:10.1016/j.jtcvs.2010.06.034
}

bypass was ischemia in the left anterior descending coronary artery territory. The left internal thoracic arterial graft was carefully examined and judged to be adequate. The etiology of the ischemia was assessed as embolic (air, atheromatous debris, or both). There was no significant valvular heart disease. The aortic valve was mildly thickened, with trace regurgitation. There was mild mitral and tricuspid regurgitation. The severe left ventricular dysfunction was managed successfully with inotropic support and intra-aortic balloon counterpulsation.

The onset of fever was first noted several hours postoperatively in the intensive care unit, with a peak of $39.6^{\circ} \mathrm{C}$. In addition to the severe fever, the postoperative course was rapidly complicated within 72 hours by depressed mental status (Glasgow coma scale sore of 6/15), sinus tachycardia (range, 140-160 beats/min), and progressive jaundice (peak serum bilirubin 3 times normal). The patient remained mildly vasodilated, with a blood pressure of $98 / 54 \mathrm{~mm} \mathrm{Hg}$ and a systemic vascular resistance in the low normal range while receiving low-dose norepinephrine. Intra-aortic balloon counterpulsation was discontinued because the cardiac index was at this point 3-fold the normal value. Postoperative transthoracic echocardiography on the fifth postoperative day demonstrated a return to the preoperative baseline, namely mild global systolic dysfunction with an ejection fraction of $45 \%$ and mild global hypokinesis.

The patient had no muscle rigidity. No source of infection could be identified. Thyroid function tests demonstrated severe hyperthyroidism with significantly elevated thyroid hormone levels and a thyroid-stimulating hormone level a 25 th of normal. A goiter was not detected on perioperative physical examination, even by the consulted endocrinologist. A very mildly enlarged thyroid was demonstrated during thyroid ultrasonography, which was performed as soon as the results of the thyroid function tests became available.

On the basis of the clinical presentation, this patient had a calculated Burch-Wartofsky thyrotoxic crisis score of 100 (a score greater than 60 is consistent with thyroid storm). ${ }^{1}$ A diagnosis of postoperative thyroid storm was made. Urgent endocrine consultation was obtained to guide emergency thyroid suppression. Therapy was commenced immediately with potassium iodide and methimazole through a nasogastric tube, as well as high-dose hydrocortisone intravenously. At this point, $\beta$-blockade was not commenced because the patient was still vasopressor dependent. $^{2}$ A significant hemodynamic response to thyroid suppression therapy was evident within 48 hours, as outlined in Table 1. The patient had strongly positive test results for antibodies to thyroid peroxidase and the thyroid-stimulating hormone receptor. This antibody profile was compatible with the diagnosis of Graves disease. 
TABLE 1. Changes in vital signs during management of thyroid storm

\begin{tabular}{|c|c|c|c|c|c|c|c|}
\hline Variable & $\begin{array}{c}\text { Preoperative } \\
\text { baseline }\end{array}$ & CTICU d 1 & CTICU d 2 & CTICU d 3 & CTICU d 4, Rx d 1 & CTICU d 5, Rx d 2 & CTICU d 6, Rx d 3 \\
\hline Temperature $\left({ }^{\circ} \mathrm{C}\right)$ & 36.8 & 39.2 & 39.6 & 39.5 & 38.5 & 38.5 & 37.1 \\
\hline Heart rate (beats/min) & 114 & 142 & 148 & 128 & 138 & 85 & 94 \\
\hline Blood pressure (mm Hg) & $85 / 56$ & $97 / 54$ & $100 / 55$ & $90 / 44$ & $117 / 46$ & $132 / 50$ & $146 / 56$ \\
\hline Norepinephrine requirement & No & Yes & Yes & Yes & Weaning begun & No & No \\
\hline
\end{tabular}

$C T I C U$, Cardiothoracic intensive care unit; $R x$, thyroid suppression therapy.

Subsequently, the potassium iodide was discontinued and the hydrocortisone was tapered during the next 5 days. The methimazole was progressively adjusted to maintenance levels on the basis of serial thyroid function tests that demonstrated a gradual return to normal. The patient's depressed mental status and jaundice also progressively resolved completely. The remainder of her hospital stay was uncomplicated. The patient remained euthyroid as an outpatient with a methimazole maintenance regimen.

\section{DISCUSSION}

Thyroid storm is a rare but life-threatening complication of thyrotoxicosis that in this case was precipitated by cardiac surgery. ${ }^{1-3}$ Thyroid storm is characterized by fever, altered mental status, gastrointestinal dysfunction, tachycardia, and high-output cardiac failure. ${ }^{1-3}$

In this report, we have described a case of thyroid storm after CABG in a 45-year-old female patient with no known history of either thyroid or coronary artery disease. Thyroid storm was diagnosed according to the Burch-Wartofsky criteria. ${ }^{1}$ This set of diagnostic criteria assesses the likelihood of thyroid storm by scoring clinical features such as body temperature, central nervous system effects, gastrointestinal or hepatic dysfunction, heart rate, heart failure, atrial fibrillation, and precipitant history. The scale ranges from 0 to 140 points, with a score greater than 60 being highly compatible with thyroid storm. ${ }^{1}$ The final diagnosis was based not only on this score but also on thyroid function tests and response to therapy.

Thyroid storm must be distinguished from other causes of hypermetabolic postoperative states, such as sepsis, malignant hyperthermia, and neuroleptic malignant syndrome. ${ }^{4,5}$ In our case, although there was no exposure to neuroleptic medications, there was intraoperative exposure to volatile anesthesia, a known trigger for malignant hyperthermia. ${ }^{4}$ The absence of muscle rigidity and hypertension, however, made malignant hyperthermia unlikely. Sepsis was unlikely as well in our case, given the onset within 24 hours after surgery and the absence of a source. Furthermore, in our case thyroid storm was suspected because of the hypermetabolic state characterized hemodynamically by tachycardia, a high cardiac output, and systemic vasodilation, requiring norepinephrine therapy. ${ }^{1-3}$ These features probably contributed to our patient's postoperative hemodynamic instability, because thyroid suppression therapy restored hemodynamic stability within 48 hours.
Thyroid storm has been reported once previously after CABG. ${ }^{6}$ Our case has multiple features in common with the previous report, such as female sex, few risk factors for coronary artery disease, minimal preoperative evidence of thyrotoxicosis, and postoperative storm complicated by coma and a prompt response to thyroid suppression therapy. ${ }^{6}$ The recognized triggers for thyroid storm include trauma, pregnancy, and infection. ${ }^{1-3}$ It is likely that the trauma of cardiac surgery triggered thyroid storm in this patient, who displayed no overt features of hyperthyroidism before the operation. This diagnosis is based on the assumption that subclinical hyperthyroidism was present before surgery, although in retrospect, the patient's tachycardia on admission may have been a sign of hyperthyroidism that was missed.

Standard therapy for thyroid storm is multimodal. ${ }^{1-3,7} \mathrm{~A}$ thioamide such as methimazole blocks the synthesis of thyroid hormone in the thyroid. Iodine therapy suppresses release of thyroid hormone through the Wolff-Chaikoff effect. Hydrocortisone therapy blocks peripheral conversion of thyroxine to triiodothyronine. In hemodynamically stable patients, $\beta$-blockade is useful to counteract the increased adrenergic tone observed in thyroid storm.

It is important to note that thyrotoxicosis may be associated with severe coronary spasm that may not necessarily resolve with aggressive medical management and may require CABG. $^{6-9}$ In our patient, there was no coronary spasm detected during coronary angiography. A probable explanation is that the non-ST elevation myocardial infarction was precipitated by the excessive myocardial oxygen demand as a result of the patient's preoperative hyperthyroidism in the setting of significant coronary stenoses. Unfortunately, we do not have preoperative thyroid function tests to support this hypothesis, but it is highly likely, as evidenced by the postoperative course. The diagnostic criteria for thyroid storm were not satisfied until after CABG had been performed.

In summary, we have described here thyroid storm after urgent CABG in a 45-year-old woman with a subsequent diagnosis of Graves disease. Although rare, this postoperative complication is important because it is life-threatening and yet eminently treatable.

\section{References}

1. Burch HB, Wartofsky L. Life-threatening thyrotoxicosis. Thyroid storm. Endocrinol Metab Clin North Am. 1993;22:263-77.

2. Redahan C, Karski JM. Thyrotoxicosis factitia in a post-aortocoronary bypass patient. Can J Anaesth. 1994;41:969-72. 
3. Klein I, Danzi S. Thyroid disease and the heart. Circulation. 2007;116:1725-35.

4. Firstenberg M, Abel E, Blais D, Andritsos M. Delayed malignant hyperthermia after routine coronary artery bypass. Ann Thorac Surg. 2010;89:947-8.

5. Mieno S, Asada K, Horimoto H, Sasaki S. Neuroleptic malignant syndrome following cardiac surgery: successful treatment with dantrolene. Eur J Cardiothorac Surg. 2003;24:458-60.

6. Lee SM, Jung TS, Hahm JR, Im SI, Kim SK, Lee KJ, et al. Thyrotoxicosis with coronary spasm that required coronary artery bypass surgery. Intern Med. 2007;47:1915-8.
7. Nayak B, Burman K. Thyrotoxicosis and thyroid storm. Endocrinol Metab Clin North Am. 2006;35:663-86, vii.

8. Choi YH, Chung JH, Bae SW, Lee WH, Jeong EM, Kang MG, et al. Severe coronary artery spasm can be associated with hyperthyroidism. Coron Artery Dis. 2005;16:135-9.

9. Patel R, Peterson G, Rohafgi A, Ghayee HK, Keeley EC, Auchus RJ, et al. Hyperthyroidism-associated coronary vasospasm with myocardial infarction and subsequent euthyroid angina. Thyroid. 2008;18:273-6.

\title{
Salvage surgery for advanced non-small cell lung cancer after response to gefitinib
}

\author{
Tomoyuki Hishida, MD, ${ }^{\mathrm{a}}$ Kanji Nagai, MD, ${ }^{\mathrm{a}}$ Tetsuya Mitsudomi, MD, ${ }^{\mathrm{b}}$ Kohei Yokoi, MD,
}

Haruhiko Kondo, MD ${ }^{\mathrm{d}}$ Hirohisa Horinouchi, MD, ${ }^{\mathrm{e}}$ Hirohiko Akiyama, MD, ${ }^{\mathrm{f}}$ Takeshi Nagayasu, MD, ${ }^{\mathrm{g}}$

Masahiro Tsuboi, MD, ${ }^{\mathrm{h}}$ and The Japan Clinical Oncology Group, Chiba, Aichi, Shizuoka, Tokyo, Saitama,

Nagasaki, and Kanagawa, Japan

Epidermal growth factor receptor (EGFR) tyrosine kinase inhibitor (EGFR-TKI) gefitinib has dramatic efficacy in more than $70 \%$ of advanced non-small cell lung cancers with EGFR gene mutations. ${ }^{1}$ Some patients with inoperable systemic non-small cell lung cancers demonstrate a downstaging of their cancer to operable disease status after gefitinib treatment. Despite high response rates for EGFR mutant tumors, the median time to progression is about 1 year. ${ }^{1}$ The EGFR T790M mutation and MET amplification are thought to be the underlying mechanisms of the acquired resistance to EGFR-TKIs. When complete resection of residual disease is possible, the patients can then be considered disease free. We have aggressively performed salvage lung resections for patients with gefitinib responses and demonstrated downstaging to NOM0. The purpose of this study was to assess

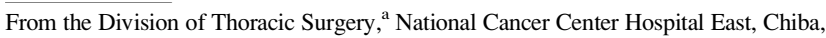
Japan; the Division of Thoracic Surgery, ${ }^{\mathrm{b}}$ Aichi Cancer Center Hospital, Aichi, Japan; the Division of Thoracic Surgery, ${ }^{c}$ Nagoya University Graduate School of Medicine, Aichi, Japan; the Division of Thoracic Surgery, ${ }^{\mathrm{d}}$ Shizuoka Cancer Center Hospital, Shizuoka, Japan; the Division of General Thoracic Surgery, ${ }^{\mathrm{e}}$ Department of Surgery, Keio University School of Medicine, Tokyo, Japan; the Division of Thoracic Surgery, ${ }^{\mathrm{f}}$ Saitama Cancer Center Hospital, Saitama, Japan; the Division of Surgical Oncology, ${ }^{\mathrm{g}}$ Department of Translational Medical Sciences, Nagasaki University Graduate School of Biomedical Sciences, Nagasaki, Japan; and the Department of Thoracic Surgery, ${ }^{\text {h }}$ Kanagawa Cancer Center Hospital, Kanagawa, Japan.

Supported in part by a Grant-in-Aid for Cancer Research from the Ministry of Health, Labour and Welfare, Japan.

Disclosures: None.

Received for publication March 4, 2010; revisions received June 13, 2010; accepted for publication June 28, 2010; available ahead of print Aug 5, 2010.

Address for reprints: Tomoyuki Hishida, MD, Division of Thoracic Surgery, National Cancer Center Hospital East, 6-5-1, Kashiwanoha, Kashiwa, Chiba, 277-8577

Japan (E-mail: thishida@nifty.com).

J Thorac Cardiovasc Surg 2010;140:e69-71

$0022-5223 / \$ 36.00$

Copyright $₫ 2010$ by The American Association for Thoracic Surgery

doi:10.1016/j.jtcvs.2010.06.035
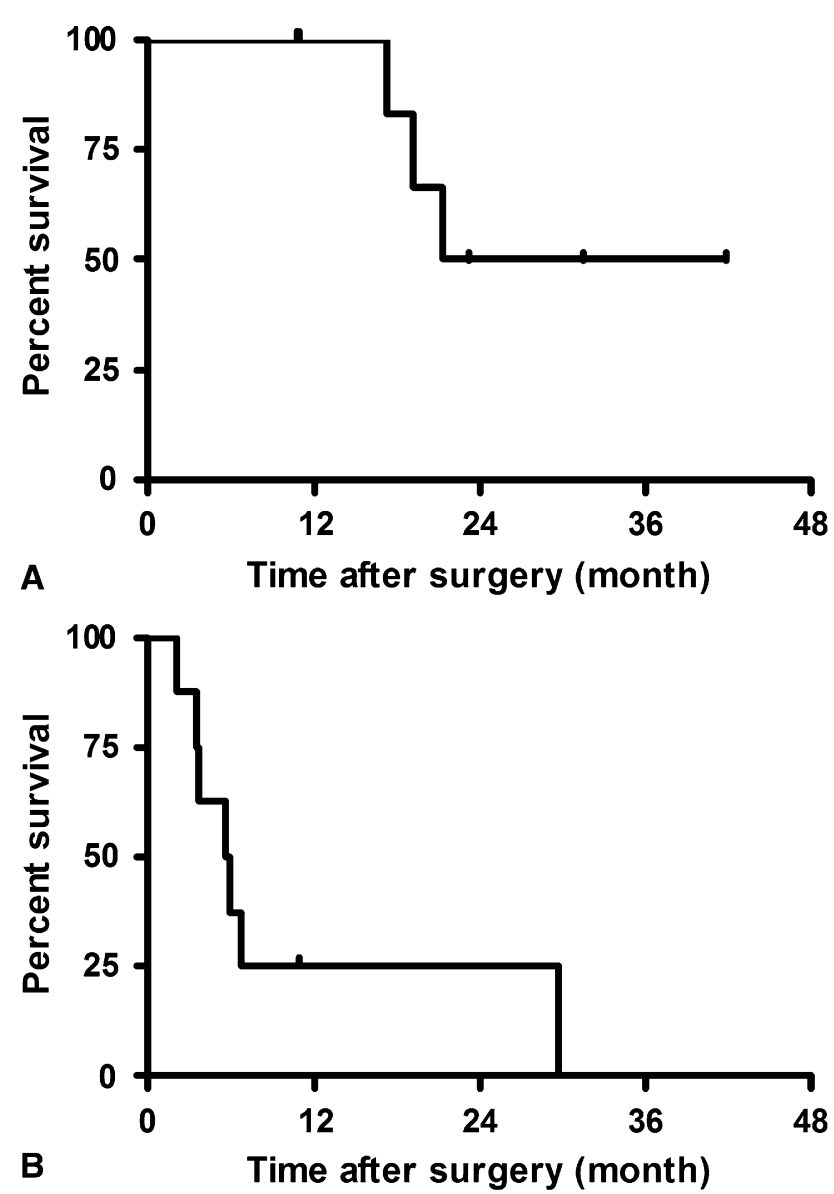

FIGURE 1. A, Overall survival curve of patients who underwent surgical resection after response to gefitinib administration. Median overall survival after surgery was 32 months. B, Recurrence-free survival curve of patients who underwent surgical resection after response to gefitinib administration. Median recurrence-free survival after surgery was 6 months. 